\title{
Phantom auditory perception (tinnitus) is characterised by stronger
} anticipatory auditory predictions

$11{ }^{1}$ Salzburg Brain Dynamics Lab, Centre of Cognitive Neuroscience, Division of Physiological Psychology, 12 University of Salzburg, Austria.

$13{ }^{2}$ Department of Otorhinolaryngology-Head and Neck Surgery, The Paracelsus Private Medical University 14 of Salzburg, Salzburg, Austria.

$15{ }^{3}$ Institute of Neuroscience, Newcastle University Medical School, Newcastle upon Tyne, United Kingdom.

$17{ }^{4}$ Department of Psychiatry and Psychotherapy, University of Regensburg, Germany.

18 Corresponding author: marta.s.partyka@gmail.com 


\section{Abstract}

How phantom perceptions arise and the factors that make individuals prone to such

21 experiences are not well understood. An attractive phenomenon to study these questions is tinnitus,

22 a very common auditory phantom perception which is not explained by hyperactivity in the

23 auditory pathway alone. Our framework posits that a predisposition to developing (chronic)

24 tinnitus is dependent on individual traits relating to the formation and utilization of sensory

25 predictions. Predictions of auditory stimulus frequency (remote from tinnitus frequency) were

26 studied using a paradigm parametrically modulating regularity (i.e. predictability) of tone

27 sequences and applying decoding techniques on magnetoencephalographic (MEG) data. For

28 processes likely linked to short-term memory, individuals with tinnitus showed an enhanced

29 anticipatory prediction pattern associated with increasing sequence regularity. In contrast,

30 individuals without tinnitus engaged the same processes following the onset of the to-be-decoded

31 sound. We posit that this tendency to optimally anticipate static and changing auditory inputs may

32 determine which individuals faced with persistent auditory pathway hyperactivity factor it into

33 auditory predictions, and thus perceive it as tinnitus. While our study constitutes a first step relating

34 vulnerability to tinnitus with predictive processing, longitudinal studies are needed to confirm the

35 predisposition model of tinnitus development. 


\section{Introduction}

Phantom perceptions do not require sensory input transduced by peripheral receptors. The common auditory phantom perception known as tinnitus affects approximately $\sim 10 \%{ }^{1,2}$ of the population. Individuals experience tinnitus by consciously perceiving relatively simple sounds such as pure tones or narrow band noises without an identifiable objective environmental or bodily source. Tinnitus can be accompanied by substantial distress and reduced quality of life, which appears to be independent of the intensity of the perceived sound ${ }^{3}$. The mechanisms by which this phantom sound emerges from ongoing brain activity (so-called "neural correlates") have still not been resolved. A broad consensus supports the idea that some form of hearing damage (with or without clear audiometric changes) ${ }^{4-6}$ stands at the outset of tinnitus development, leading to maladaptive functional or structural changes within or beyond the auditory system ${ }^{7-9}$. By far the most popular view postulates a change of neural gain in deprived regions of the auditory pathway, thereby amplifying spontaneous activity which is interpreted as sound by downstream cortical regions (for review see ${ }^{10}$; we will subsequently refer to this general idea as altered gain model).

Research along these lines has focused mostly on probable "neural correlate" candidates

of tinnitus such as increased spontaneous firing rate or enhanced neural synchrony. The altered gain model of tinnitus is substantially supported by studies in animals ${ }^{11}$, despite the obvious challenges in obtaining subjective reports. In humans the supporting evidence for this model is less apparent, partly because (contrary to animal models) the research is focused on chronic rather than acute tinnitus, but also due to a lack of understanding as to how measures commonly obtained in humans (such as oscillatory power in M/EEG or BOLD in fMRI) can be translated to those used to support the altered gain model. Based on human and animal works in other domains ${ }^{12}$, reduced ongoing alpha or increased gamma in auditory regions pertinent for phantom sounds (for other auditory phantom percepts see ${ }^{13,14}$ ) may be relevant to perception of tinnitus. However, the empirical evidence is inconclusive ${ }^{15,16}$. With the exception of technical or practical issues that may complicate a convincing confirmation of the altered gain model in humans, other observations speak in favour of its explanatory insufficiency ${ }^{17}: 1$ ) Only a fraction of individuals who suffer a hearing impairment will experience tinnitus $\left(\sim 70 \%\right.$ following sensorineural hearing loss; see ${ }^{18-}$ $\left.{ }^{20}\right)$. 2) The onset of tinnitus and the onset of the hearing loss often occur at different times. 3) Not all cases of acute tinnitus will become chronic. One possibility to overcome these explanatory gaps is to frame tinnitus perception within a Bayesian inference framework ${ }^{21}$, which emphasizes the constructive nature of perception being guided by internal models ${ }^{22}$. In order to establish and

72 improve internal models, incoming sensory input is compared to predictions (so-called priors), 
73 which need to be cast in real-time in dynamic environments. In a recent predictive coding view,

74 tinnitus is seen as a consequence of a default prediction of silence altering to one of sound when

75 faced with (enhanced) spontaneous activity ("tinnitus precursor") along the auditory pathway ${ }^{21}$.

76 While conceptually overcoming many inconsistencies related to the altered gain model ${ }^{17}$, strong

77 support for this view is lacking partially due to the non-trivial task of deriving robust and direct

78 measures of tinnitus-supporting priors from ongoing brain activity. Recent work has found indirect

79 evidence of altered priors in established tinnitus ${ }^{23}$, but the question of how and why such altered

80 priors should even emerge in certain individuals remains open.

81 A recent line of reasoning holds that increased precision of priors could drive hallucinatory

82 experiences ${ }^{24,25}$. Indeed, interindividual variability in prior strength assessed in a visuo-auditory

83 conditioning task predicts the experience of hallucinations in daily life ${ }^{26}$. We postulate that the

84 predisposition to developing tinnitus may be contingent on an individual's - putatively relatively

85 stable, "trait-like" - tendency to more strongly engage in predictive processing in the auditory

86 modality. Ideally individualized measures of auditory predictive processing tendencies would be

87 obtained before a potentially tinnitus-inducing event and then compared between individuals that

88 do or do not develop (chronic) tinnitus. However, this is difficult to pursue in humans for ethical

89 and practical reasons. In a first step to establish our tinnitus-predisposition framework, we focus

90 on comparing individuals with chronic tinnitus and healthy controls. Using stimulus frequencies

91 remote from those of tinnitus should reduce the chance of identifying consequences rather than

92 causes of tinnitus.

93 Our hypothesis implies that when processing auditory input, individuals with tinnitus

94 should engage predictions more strongly, that is, either more accurately or anticipatory, compared

95 with individuals without tinnitus. Recently we established a powerful experimental approach 27

96 showing in normal hearing individuals that more regular pure tone sequences activate

97 tonotopically specific auditory templates in an anticipatory manner (see ${ }^{28,29}$ for similar findings

98 in the visual modality). In line with our predisposition framework, with increasing statistical

99 regularities of sound sequences, individuals with tinnitus exhibited stronger anticipatory

100 representations of upcoming stimuli.

102 Results

$103 \quad 34$ individuals with chronic tinnitus (16 females) took part in the experiment. For 25

104 individuals in the Tinnitus group, age-matched volunteers without tinnitus (17 females) were 
105 recruited for the purpose of group comparisons. Magnetoencephalography (MEG) was used to

106 record neural activity while participants passively listened to sequences composed of pure tones

107 at four different carrier frequencies. High temporal expectation was ensured by a strict rhythmic

108 presentation at $3 \mathrm{~Hz}$. While sound onsets were perfectly predictable, the probability of which

109 carrier frequency would be presented (and thus could be predicted) was varied by parametrically

110 modulating the regularity (i.e. predictability) of sound sequences across conditions (see Figure 1a

111 and Methods for details). To investigate feature-specific predictive auditory processing also in

112 absence of stimulation, sounds were omitted randomly in $10 \%$ of presentations. Tinnitus

113 characteristics and tinnitus-related distress were assessed with online versions of standardised

114 questionnaires (see Methods for details) shortly prior to the visit to the laboratory.

115 To measure the dynamics of auditory predictions we used multivariate pattern analysis

116 (MVPA) to derive feature (carrier frequency) specific information from the MEG data. Following

117 our previous study ${ }^{27}$, we trained classifiers to temporally decode the carrier frequency presented

118 in the random sound sequence. These trained classifiers were subsequently tested on sound events

119 in all regularity levels using time- and condition-generalization ${ }^{30}$. For each individual we

120 quantified how decoding accuracy was modulated by the regularity condition by extracting the

121 slope ( $\beta$ coefficients) from a linear regression analysis. These were compared between the groups,

122 yielding a time-generalized representation of T-values (see Figure 1).

123

124

125

126

127 
bioRxiv preprint doi: https://doi.org/10.1101/869842; this version posted December 10, 2019. The copyright holder for this preprint (which was not certified by peer review) is the author/funder, who has granted bioRxiv a license to display the preprint in perpetuity. It is made available under aCC-BY-NC-ND 4.0 International license.

a)

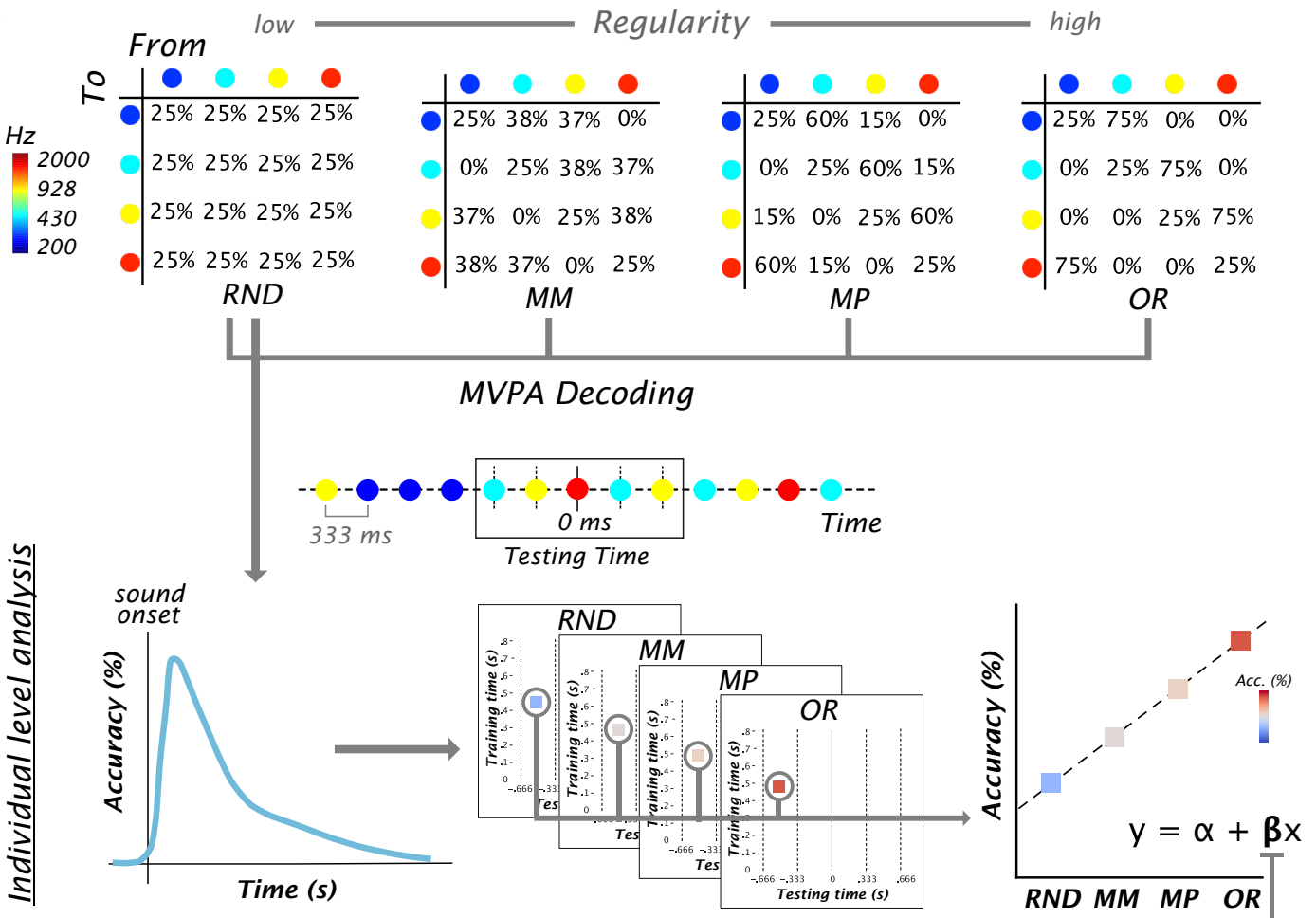

c)

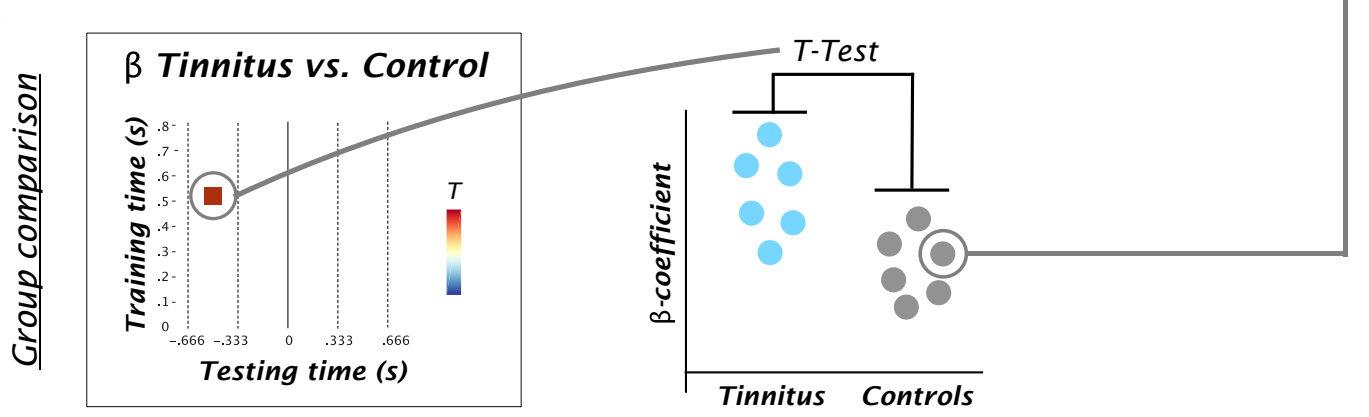

Figure 1: Experimental design and analysis rationale. a) Transition matrices used to generate sound sequences according to the different conditions (random [RD], midminus [MM], midplus [MP] and ordered [OR]) with a schematic example of a brief sound sequence. $10 \%$ of sound stimuli were randomly omitted. The "Testing Time" window corresponds to one trial with the to-be-decoded carrier frequency in the center (at $0 \mathrm{~ms}$; marked by solid line), preceded and followed by two other tones (marked by dashed lines). b) For MVPA, time-shifted classifiers were trained on events in the random condition (left panel) and applied in a condition- and timegeneralized manner to all conditions (middle panel). For every time-generalized data point, the dependence of decoding accuracy on the regularity of the sound sequence was quantified by a linear regression. $c$ ) At a group level, the resulting slopes ( $\boldsymbol{\beta}$-coefficients) of the regression analysis were compared between the tinnitus group and the control group.

Normal neural encoding of carrier frequencies in tinnitus

Sensor level MEG data was used to decode the four carrier frequencies presented in the random sound sequence (Figure 1a and $\mathbf{b}$ ). The trained classifiers were fundamental for targeting

142 the main question of whether feature specific predictions in the auditory system are engaged

143 differently in each of the groups in all further steps. In a first step, we could analyse the results of

144 the simple decoding analysis for the random condition. Since this condition did not contain

145 predictability-related information it allowed us to compare basic encoding of sound carrier 
146 frequencies in individuals with tinnitus with the control group. Both groups exhibited a rapid 147 increase of decoding accuracy following sound onset robustly observed at an individual level

148 (Figure 2a). Above chance ( $p<.05$, Bonferroni corrected) decoding accuracy started immediately 149 after stimulus onset in both samples (note that sampling rate was at $100 \mathrm{~Hz}$ ). While peak increases 150 were reached at approximately $100 \mathrm{~ms}$, decoding accuracy remained statistically significant above 151 chance for approximately $\sim 500-600 \mathrm{~ms}$ with some interindividual variability. Remarkably, given 152 the passive and non-engaging nature of the experiment, this means that carrier frequency specific 153 information remained available during the two subsequent sound presentations. Interestingly, 154 accuracy transiently increased approximately $100 \mathrm{~ms}$ after the subsequent stimulus onset (i.e. 450$155500 \mathrm{~ms}$ after the to-be-decoded sound). Descriptively a similar pattern was observed following the 156 next but one stimulus, albeit at a much smaller magnitude. These observations may reflect a 157 sustained activation and reactivation of an auditory short-term memory trace enabling the 158 formation of associations between events in temporal proximity, which is fundamental for 159 subsequent learning of statistical regularities.

160 Importantly, we found no differences between the tinnitus group and the control group 161 when carrier frequencies were presented randomly (Figure 2a). Since the upper carrier frequency 162 of $2 \mathrm{kHz}$ was at or below the audiometric edge for the majority of individuals with tinnitus (see 163 audiograms in Supplementary Material Appendix 1 and 2), superior decoding results could 164 plausibly be expected in the case of an enlarged neural representation of non-deprived tone 165 frequencies resulting from tonotopic changes ${ }^{7}$. Given the presence of hearing loss and potential 166 tonotopic reorganization in individuals with tinnitus, the absence of a group difference in this 167 simple carrier frequency decoding is of outstanding importance: that is, at a basic level individuals 168 with tinnitus encode carrier frequencies equally well to individuals without tinnitus. This means 169 that subsequently reported group differences are due to the manipulation of regularity (i.e. 170 predictability) of the sound sequence. 
bioRxiv preprint doi: https://doi.org/10.1101/869842; this version posted December 10, 2019. The copyright holder for this preprint (which was not certified by peer review) is the author/funder, who has granted bioRxiv a license to display the preprint in perpetuity. It is made available under aCC-BY-NC-ND 4.0 International license.

a) Carrier-frequency decoding in random sequence

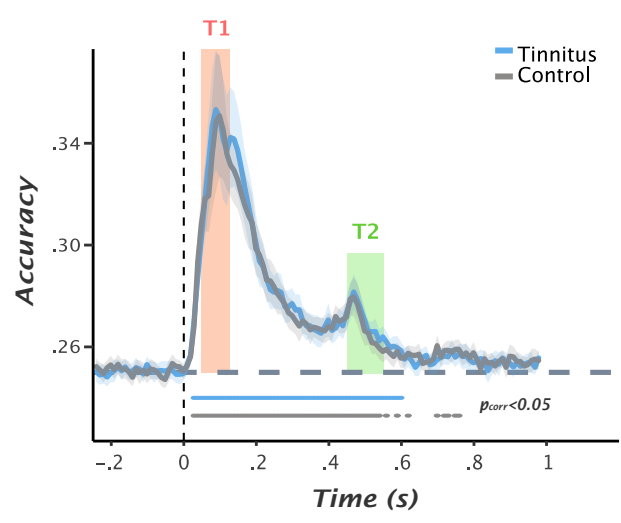

c)

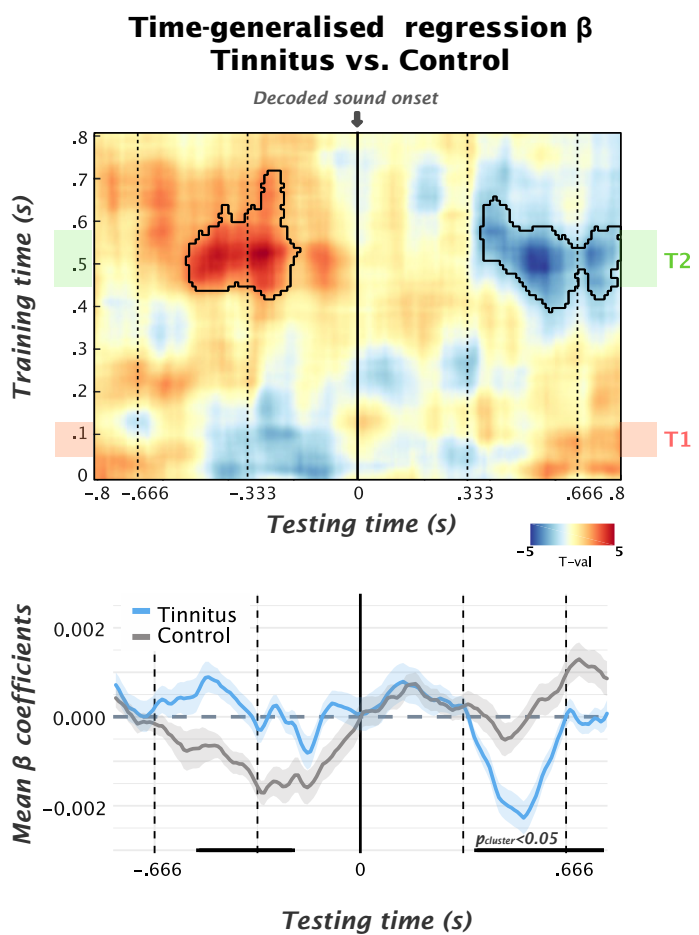

b)

\section{Informative activity}
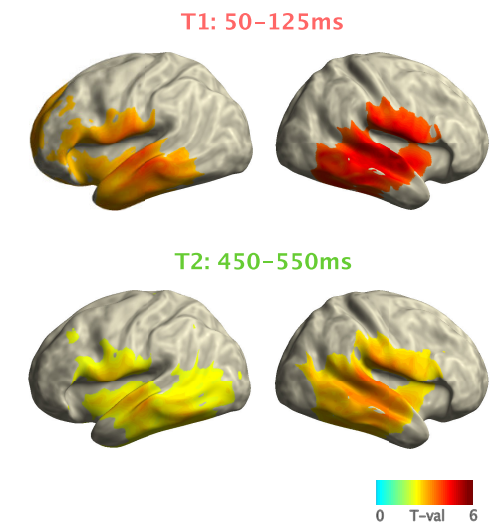

d)

Individual level results

Pre-stimulus testing time

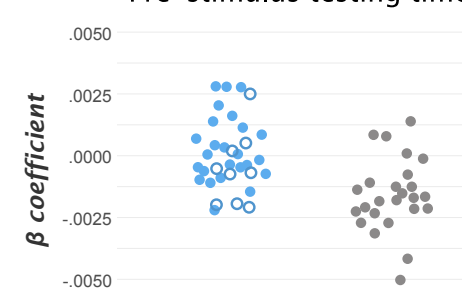

Tinnitus Controls

Post-stimulus testing time

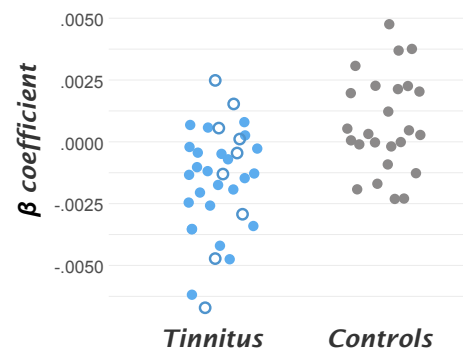

Figure 2. a) Temporal decoding of carrier frequencies in the random sound sequence for the tinnitus and control groups, respectively. In both groups, peak accuracy is reached after $\sim 100 \mathrm{~ms}$ following sound onset. Above chance decoding accuracy is observed in a sustained manner up to $\sim 600 \mathrm{~ms}(p<.05$, Bonferoni corrected). No differences were observed between the groups. b) Source level depiction of Informative Activity for different periods: $50-125 \mathrm{~ms}$ (T1) and 450-550ms (T2) after decoded sound presentation. The latter corresponds to the training time interval yielding pronounced group differences in the condition generalized analysis. c) (upper panel) Group comparison (see Figure 1c) of $\beta$-coefficient values between tinnitus vs. control groups in time-generalised matrix. Colors indicate t-values and solid black borders delimiting periods of significant difference $(p<0.05$, cluster corrected). Lower panel: Time courses of $\beta$-coefficients averaged over $480-580$ ms training time-window, showing aforementioned effects driven by a relative increase of regularity-dependent carrier frequency specific activity prior to anticipated onset period and downregulation in the postsound period in the tinnitus group. d) For illustration purposes, individual $\beta$-coefficient values within pre- and post-sound cluster are shown. While for the group comparison (shown in c) a subset of 25 individuals with tinnitus were taken into account, the full sample of 34 participants with tinnitus is displayed (individuals not considered in group comparison shown as hollow circles). 


\section{Regularity-driven carrier frequency specific neural information strongly differs between tinnitus and control groups}

To adequately capture carrier-frequency specific, predictive-processing dynamics, we used

192 a classifier trained on the random sound sequence (shown above) and applied it to all regularity

193 levels in a time-generalized manner (Figure 1a). We used decoding accuracy as an indicator of

194 the strength of internal representation of the particular stimulus frequency, and thus as a window

195 into its utilisation in predictive processes. In order to quantify how the predictability of the carrier

196 frequency modulates corresponding neural information, for each individual we calculated linear

197 regressions (at each time-point over the entire temporal generalization matrix) between decoding

198 accuracy and increasing regularity level (Figure 1b). In both groups, for the early training-time

199 periods $(\sim 50-350 \mathrm{~ms})$, similar patterns - in particular the anticipatory pre-activation of carrier

200 frequency specific neural templates - were revealed as in the original experiment despite the

201 slightly different analysis approach (see Supplementary Material Figure S3). For each point in the

202 time-generalization matrix we compared the individual $\beta$-coefficients between groups using a $t$ -

203 test, reflecting differences in how carrier frequency specific predictions are modulated by the 204 regularity of the sequence (Figure 1c).

205 Striking effects were obtained for relatively late training time intervals centred at around $530 \mathrm{~ms}$. For trials in which the decoded sound was presented at testing time 0 (Figure 2c), we identified a positive cluster $(p=0.038)$ prior to the onset of the to-be-decoded event at

208 approximately $-530 \mathrm{~ms}$ to $-200 \mathrm{~ms}$, indicating a relatively stronger increase of decoding accuracy 209 with regularity level for individuals with tinnitus. We interpret this as evidence of stronger correct 210 anticipation of the present stimulus by individuals with tinnitus, in the higher regularity conditions

211 where such anticipation is possible. We observed a similar effect in omission trials (see

212 Supplementary Materials Figure S5). Time courses of $\beta$-coefficients averaged over the relevant

213 late training time period (Figure 2c) showed that the intergroup differences were driven by

214 opposing patterns: whereas individuals with tinnitus exhibited relatively increased carrier

215 frequency specific information with stronger predictability prior to anticipated sound onsets,

216 results for control individuals were marked by an augmenting absence (captured by the negative

$217 \beta$-coefficients) of the carrier frequency pattern anticipated at $0 \mathrm{~ms}$. Following the sound onset, a

218 negative cluster $(p=0.05)$ between $360 \mathrm{~ms}$ and $800 \mathrm{~ms}$ was observed for the same training time

219 interval. Similar to the prestimulus results, these post-sound onset effects are caused by inverse

220 tendencies for the tinnitus and control groups (Figure 2c): that is, whereas individuals with tinnitus

221 appeared to quickly deactivate carrier frequency patterns the more regular the sound sequence 
222 became, control individuals reactivate patterns of the decoded sound presented at 0 ms upon

223 presentation of new events.

224 In order to make sense of this seemingly complex picture, it is important to detail the

225 stimulation structure in light of our analysis approach, which focused on representation of the

226 present stimulus frequency presented at time 0 . Differing sequence regularities did not change the

227 probability of the stimulus frequency remaining the same from one stimulus to the next (fixed at

2280.25 ; i.e. diagonal of transition matrix), but increasing stimulus regularity did reduce the

229 probability of the stimulus frequency remaining the same over separations of two or more stimuli.

230 The observed regularity-related differences occurring from around two or more stimuli prior or

231 subsequent to the present stimulus can be reconciled with the fact that relatively late training-time

232 neural patterns capture this group-level effect. These patterns likely reflected processes associating

233 sequential inputs, that is, short-term memory processes that integrate information over longer

234 timescales. Our results suggest that in highly predictable sequences, control individuals engage

235 these feature-specific auditory short-term processes in a more reactive way. Qualitatively this is

236 similar to the manner they are activated in random sequences, that is, the stimulus that has just

237 been heard is continuously represented and reactivated when new input arrives. Tinnitus

238 individuals on the other hand exhibit a rather proactive engagement of the same processes with

239 increasing regularity, preactivating stimulus representations in auditory short-term memory before

240 their actual onset. Upon presentation of subsequent stimuli - which become less likely to be the

241 same carrier frequency as presented at 0 - feature-specific neural patterns are downregulated.

242 Overall, our results point to a dramatically altered involvement of higher level auditory short-term

243 memory processes related to associating discrete events to form representations ("internal

244 models") of the statistical regularity of the sound sequence. These findings support the hypothesis

245 that individuals with tinnitus utilize internal models in a more anticipatory manner when

246 processing auditory events.

\section{Regularity-dependent engagement of internal models is unrelated to the magnitude of} hearing loss and subjective tinnitus features

Following the demonstration of a marked group difference in activating late carrier

251 frequency-specific neural patterns as a function of sequence regularity, we tested whether the 252 magnitude of this process was related to subjectively rated tinnitus characteristics as well as 253 audiometric features. Across the full $(N=34)$ tinnitus sample, we performed Spearman correlation 254 between the averaged $\beta$-regression values corresponding in time to statistically significant 
anticipatory positive and post-stimulus negative clusters and magnitude of hearing loss (HLS, measured by Tinnitus Questionnaire ), tinnitus loudness (TL) and tinnitus distress (TD) (see Supplementary Material Figure S5).

In spite of the explorative (liberal) testing without multiple comparison corrections, no significant correlation effects for any of these factors were identified for the prestimulus positive cluster ( HLS: rho $=-0.6, p=0.75$; TL: $r h o=-0.06, p=0.73$; TD: $r h o=0.11, p=0.53$ ) nor for the post-stimulus negative effect ( HLS: $r h o=-0.13, p=0.45$; TL: $r h o=-0.01, p=0.95$; TD: rho $=-0.14, p=0.43$ ). The lack of relationships between prediction related neural effects with hearing loss add further support to the claim that the effects visible in group analysis are strictly regularitydependent and not driven by low-level auditory processing. From a "neural correlate" perspective, the lack of correlation with tinnitus-specific (distress and loudness) measures would seem counterintuitive. However, this result is fully compatible with the predisposition view that we are advancing, proposing that individual predictive processing tendencies are relevant for the emergence and stabilization of tinnitus.

\section{Discussion}

Current "neural correlate"-based approaches of tinnitus are insufficient to explain the interindividual varying trajectories that lead some individuals to develop (chronic) tinnitus following hearing damage but not others. Our predictive processing predisposition framework relies on inter-individual trait differences in applying internal models in the auditory system. Vulnerability to developing (chronic) tinnitus may arise from stronger tendencies to process incoming sounds according to internal model-based predictions: These tendencies could both refer to absolute strength (precision) or altered temporal dynamics (i.e. becoming more anticipatory) of auditory predictions. The individual's predictive processing tendency could lead to different clinical outcomes when faced with potentially tinnitus-inducing events such as increased spontaneous activity and/or synchrony in the auditory pathway that follows hearing damage or noise overexposure. For instance, individuals better able to predict the dynamics of this spontaneous activity over time would form stronger predictions of it, thus facilitating its perception as an auditory entity through altered predictions ${ }^{21}$. However, other frameworks that emphasize the importance of top-down control of auditory activity to play a role in tinnitus generation (e.g. ${ }^{31}$ ) are also compatible with our predisposition concept. In a first necessary step towards establishing support for this novel framework, we compared individuals with chronic tinnitus and controls 
287 without tinnitus, utilizing an approach ${ }^{27}$ that allows us to scrutinize feature-specificity of 288 predictive processes in the auditory system at high temporal resolution. In contrast to "neural 289 correlate" approaches, no special importance was placed on the tinnitus frequency. Our main 290 findings are: 1) basic processing of carrier frequencies is not altered in tinnitus; 2) higher-level 291 (short-term memory-based) processing of carrier frequency exhibit a stronger anticipatory pattern 292 in individuals with tinnitus as compared to controls; 3 ) the latter pattern is not correlated to factors 293 such as magnitude of hearing loss or tinnitus-related variables (distress and loudness), in line with 294 the idea that they reflect a more general predictive processing tendency of the individual.

296 of predictability (set by the regularity of the sequence) used training classifiers to decode carrier 297 frequencies in the random sound sequence. While our framework would predict strongest 298 differences in situations when reliable internal models can be formed, it was important to also 299 scrutinize processing of carrier frequencies when precise predictions cannot be made. Differences 300 could be plausibly expected since most individuals with tinnitus exhibit some hearing loss at higher 301 frequencies putatively leading to cortical reorganization: In particular an expanded representation 302 of non-damaged cochlear regions ${ }^{7}$ and potential improved sensory processing thereof ${ }^{32}$ could 303 imply an improved decoding performance in the random sequence. However, the temporal 304 decoding patterns were virtually identical for both groups, with the characteristic features 305 elaborated on in our previous report ${ }^{27}$ (e.g. the rapid onset and relatively sustained above-chance 306 decoding performance outlasting subsequent tone presentations). The lack of a group difference is 307 overall in line with findings indicating no abnormal tonotopic representation in tinnitus ${ }^{33}$ in 308 contrast to earlier reports ${ }^{34}$. Making a stronger point on this issue would require establishing that 309 decoding performance in the random sequence can be taken as a quantitative proxy for tonotopic 310 representation. Importantly for the current study, all group differences we reported result not from 311 low-level, feedforward activation of tonotopically neural ensembles, but from adding varying 312 levels of regularity to the sound sequence.

313 Indeed, striking regularity-dependent group differences were observed, with rich temporal 314 information that can only be uncovered using high-temporal resolution methods: Firstly, while the 315 general peak of decoding accuracy occurred at $100 \mathrm{~ms}$ and in these early training-time windows 316 exhibited a positive relationship with regularity (see Figure S3 in Supplementary materials) as 317 described in ${ }^{27}$, these early periods did not capture group differences. For late training-time 318 intervals, however, marked group differences were observed. Interestingly, the relevant training 319 time interval is $\sim 150 \mathrm{~ms}$ after the onset of the sound following the to-be-decoded sound. This late 
320 increased accuracy for decoding carrier frequencies in the random sequence indicates a

321 reactivation of a short-term memory representation of carrier frequency specific information

322 presented at $0 \mathrm{~ms}$ (see also the descriptive similarity of Informative Activity patterns for early and

323 late periods in Figure $\mathbf{2 b}$ ). This process leads to a co-activation of new with previous input, which

324 is crucial for associating discrete events via Hebbian principles. These learned associations are

325 crucial for building up an internal model of the statistical regularities underlying the generation of

326 the sound sequence. The selective involvement of these late processes in terms of group differences

327 points to the role of high-level (memory based) auditory processes contributing to (or

328 predisposing) tinnitus beyond purely bottom-up driven processes. An open question however

329 remains as to whether these differences would be seen without the reactivation caused by a

330 subsequent sound. A study systematically varying the ISI would be needed to resolve this issue,

331 showing whether the latency of effects would remain relatively stable or follow the temporal

332 separation of events.

333 Secondly, the temporal resolution of MEG allowed us to precisely describe the temporal

334 dynamics of how these higher level auditory processes are engaged in the context of different

335 levels of regularity of the sound sequence and how they differ between the groups (Figure 2c).

336 Effects were dependent on whether the time-window of investigation (testing time) was prior to or

337 following the onset of the to-be-decoded sound (testing time at $0 \mathrm{~ms}$ ). Both groups showed an

338 immediate engagement of these short-term memory-related auditory processes following the

339 (perfectly predictable in time) sound onset. At later intervals ( $\sim 600 \mathrm{~ms})$, coinciding with the onset

340 of the second sound following the to-be-decoded sound, decoding accuracy increased in the control

341 group with increasing regularity of the sequence (see Figure 2c). The pattern was reactive in the

342 sense that in periods prior to the anticipated onset of the to-be-decoded sound, carrier frequency

343 specific information was less present with increasingly regular sounds. This indicates that short-

344 term memory related auditory processes are engaged only once a predicted sound is presented,

345 potentially contributing to a continuous update and stabilization of a formed internal model.

346 Individuals with tinnitus, however, show an almost mirror-image pattern to the control group, with

347 stronger anticipatory engagement of short-term memory related auditory processes when the

348 sequence becomes more regular. Following the anticipated onset of a more predictable sound

349 (carrier frequency) a marked disengagement of the relevant carrier frequency specific neural

350 patterns is observed: this could be partially driven by processing the sound presented at $333 \mathrm{~ms}$ or

351 anticipating the sound presented at $666 \mathrm{~ms}$, both (usually) differing from the one presented at $0 \mathrm{~ms}$

352 in regular sequences. Irregardless, the results point to a dramatic difference with respect to internal 
models utilization between individuals with tinnitus and the control group. Overall, the more anticipatory pattern in tinnitus is in line with our belief that stronger predictive processing tendencies could identify individuals vulnerable to developing tinnitus. On a broader level the observed effects are also in accord with reports linking strong priors to general proneness to auditory hallucinations, even though a link between our data and those derived from computational modeling of behavioral data would need to be established. Also in contrast to a previous study supporting this notion ${ }^{26}$, we derive our conclusions from neural data obtained during passive sound processing without experimentally inducing illusory percepts. The simplicity of our approach may be useful for studying altered predictive processing in other clinical groups, including ones in which behavioral assessment is challenging ${ }^{35}$.

Albeit striking in terms of strength, the group effects reported here do not conclusively confirm a core idea that we are advancing, namely that increased internal model utilization tendencies in the auditory system predispose development of tinnitus. The absence of correlations with variables associated with tinnitus-induction (e.g. hearing loss) or consequences of tinnitus (e.g. loudness or distress), supports the view that the predictive processes we observe using our approach could be a temporally more stable "trait-like" feature of the individual. However, strong evidence would ultimately require longitudinal studies in humans ideally starting measurements prior to onset of (chronic) tinnitus, which is challenging (for an approach to inducing transient tinnitus see ${ }^{4}$ ). Thus a next step may be to apply this paradigm in animal models of (chronic) tinnitus, where inter-animal variability has also been reported (e.g. ${ }^{36}$ ). Such an approach should

373 be relatively straightforward since the paradigm does not require any task for which the animal

374 needs to be trained. Also when neural recording is performed using multiple electrodes, large parts 375 of the analysis described here could be applied.

To summarize, we show for the first time enhanced anticipatory engagement of featurespecific high-level (putatively short-term memory based) predictive auditory processing in individuals experiencing chronically auditory phantom perception - tinnitus. However, whether this pattern constitutes a predisposing factor or is a consequence of tinnitus onset (despite being uncorrelated to tinnitus-relevant features) remains to be addressed in future studies. Resolving this 381 issue has far-reaching consequences on a conceptual level by narrowing the explanatory gap of 382 who will develop tinnitus following hearing damage. Also on a clinical level our work could have 383 important implications, by potentially being able to identify individuals with greater risk of 384 developing (chronic) tinnitus, thereby enabling more focused prevention or treatment efforts. 
Materials and methods

Participants

A total of 34 individuals with tinnitus (17 females, 20-67 years old, mean age $=45.12$, $s d=13.65$ ) participated in the experiment: 25 (16 females, 20-66 years old, mean age $=40.92$, $s d=13.17$ ) were age-matched (in all cases but one both age- and sex- matched) with the control group and used for group comparisons. Tinnitus related questionnaires (German version of Tinnitus Questionnaire, TQ; ${ }^{37}$, Tinnitus Sample Case History Questionnaire, $\mathrm{TSCHQ}^{41}$ and 10point scale Tinnitus Severity, TS) were collected for individuals with tinnitus. Standardized puretone audiometric testing for frequencies from $125 \mathrm{~Hz}$ to $8 \mathrm{kHz}$ was performed in 31 out of 34 tinnitus participants using Interacoustic AS608 audiometer. 25 volunteers (17 females, 21-65 years old, mean age $=41.56, s d=13.68)$ reporting no relevant audiological, neurological or psychiatric treatment history took part as a control group. 12 of the group were part of an experiment published elsewhere ${ }^{27}$. Control subjects were age-matched to each tinnitus participant by the $+/-3$ years criterion, selecting the closest match in cases where more than one subject was eligible. No differences were shown for age between the samples comprised in the intergroup analysis $(t=$ $0.17, p=0.89)$. All participants provided written informed consent prior to participating. The experimental protocol was approved by the ethics committee of the University of Salzburg (EKGZ: 22/2016 with Addenda).

\section{Stimuli and experimental procedure} entering the MEG shielded chamber. The Polhemus FASTRAK (Polhemus, Colchester, Vermont, U.S.A) digitizer was used to digitize head shape and position for each individual via marking of anatomical references (nasion and left/right pre-auricular points), location of HPI coils and approximately 300 additional points over the scalp. Before the start of the actual paradigm, a 5 min resting state recording was performed (not reported here), when subjects were asked to simply look at the center of the rear-projection screen.

During the experiment, participants watched a silent movie ("Cirque du Soleil: Worlds

415 Away"), while passively being exposed to different tone sequences (Figure 1a). No instruction 416 considering the sound stimuli was provided. The movie was displayed on the screen inside the 417 shielded room using a projector (PROPIXX, VPixx technologies, Canada) and a periscope, 
418 whereas auditory stimulation was delivered to both ears via MEG-compatible pneumatic in-ear

419 headphones (SOUNDPixx, ibid). Four different pure (sinusoidal) tones were presented, with

420 carrier frequencies logarithmically spaced between 200 to $2000 \mathrm{~Hz}(200 \mathrm{~Hz}, 431 \mathrm{~Hz}, 928 \mathrm{~Hz}, 2000$

$421 \mathrm{~Hz}$ ). Each of the tones lasted $100 \mathrm{~ms}$, tapered with $5 \mathrm{~ms}$ linearly ascending/descending periods at

422 both ends. Sounds were presented at a constant $3 \mathrm{~Hz}$ stimulation rate.

423 Each participant was presented four blocks of tone sequences comprising 4000 stimuli,

424 each lasting approximately 22 mins. The number of particular tone frequencies was balanced

425 across blocks, so the condition-blocks varied solely by presentation order, which was 426 parametrically modulated in their regularity (entropy) level using different transition matrices ${ }^{42}$.

427 In the random condition (RD, highest entropy or lowest regularity; see Figure 1a) there was an 428 equal transition probability from one sound to another (thus preventing any possibility of 429 accurately predicting an upcoming stimulus). Conversely, in the ordered condition (OR, lowest 430 entropy level or highest regularity), presentation of one sound was for the majority (75\% of cases) 431 systematically followed by the particular other sound. Additionally, two intermediate entropy 432 conditions were included, labelled here as midminus (MM) and midplus (MP). To control for the 433 influence of self-repetitions, the diagonal of the transition matrices was set to be always $25 \%$ across 434 all entropy conditions. The experiment was written using the MATLAB (ver. 9.1 The MathWorks, 435 Natick, Massachusetts, U.S.A) based Psychophysics Toolbox ${ }^{43}$.

\section{MEG data acquisition and preprocessing}

438 Brain magnetic activity was measured using a whole-head MEG (Triux, MEGIN Oy, 439 Finland), sampling the signal at $1000 \mathrm{~Hz}$ and with the default hardware filters set by the 440 manufacturer $(0.1 \mathrm{~Hz}$ high pass - $330 \mathrm{~Hz}$ low pass). Subjects were comfortably seated inside a 441 dimly lit magnetically shielded room (AK3b, Vacuumschmelze, Germany). Signals were captured 442 by 102 magnetometers and 204 planar gradiometers placed in 102 different positions. We used a 443 signal space separation algorithm $\left(\mathrm{SSS}^{44}\right)$ implemented in the Maxfilter program (version 2.2.15) 444 to attenuate external noise from the MEG signal (mainly $16.6 \mathrm{~Hz}$, and $50 \mathrm{~Hz}$ plus harmonics) and 445 realign data to a common standard head position (“-trans default” Maxfilter parameter) across 446 different blocks based on the measured head position at the beginning of each block ${ }^{45}$. The rest of 447 the subsequent analysis was performed on magnetometers only, given the mixing of information 448 between the two sensors types after the Maxfilter step ${ }^{46}$. 
Data analysis was carried out with scripts written in-house, using the Fieldtrip toolbox 47 (git version 20170919). First a high-pass filter at $0.1 \mathrm{~Hz}$ (6th order zero-phase Butterworth filter) was applied to the raw data. Then, the continuous data were chunked in $10 \mathrm{~s}$ blocks, down-sampled to $256 \mathrm{~Hz}$, and used as input to an Independent Component Analysis (ICA) algorithm. The ICA components were visually inspected to find eye blinks, eye movements, heartbeat and $16^{2} / 3 \mathrm{~Hz}$

454 (German/Austrian train power supply) artifacts. Finally, the continuous data were epoched from 1

$455 \mathrm{~s}$ before to $1 \mathrm{~s}$ after target sound/omission onset and the artifactual components projected out (mean

$4563.6 \pm 1.2 \mathrm{SD}$ ) components removed on average per each subject). All trials were kept using these 457 preprocessing steps ${ }^{45}$. A further $30 \mathrm{~Hz}$ low pass filter (6th order zero-phase Butterworth filter) and $458100 \mathrm{~Hz}$ resampling were applied to the epochs, before continuing with the multivariate pattern 459 analysis (MVPA).

\section{Multivariate Pattern Analysis (MVPA) and classifier weights projection.}

We used MVPA as implemented in the MVPA-Light (https://github.com/treder/MVPALight, commit 003a7c), forked and modified in order to extract the classifier weights (https://github.com/gdemarchi/MVPA-Light/tree/devel). In essence, we implemented the analysis of carrier frequency decoding separately for sound and omission trials (sound-to-sound decoding and sound-to-omission decoding, respectively). We defined four targets (classes) for the decoding related to the carrier frequency of the sound presented in each trial. In order to focus solely on neural templates corresponding to carrier frequency-related information and avoid any potential carry over effect from the previous sound, the classifier was trained only on the random (RD) sounds and the preceding tone frequencies were balanced across trials. The exact details of the MVPA analysis have been described elsewhere ${ }^{25}$. An identical procedure was applied to soundto-omission decoding (see Figure S4 in Supplementary materials). We trained a multiclass LDA classifier on each sample point of the random (RD) condition and tested on all regularity level conditions for each time point of the testing set using a temporal generalization method ${ }^{30}$. This enabled classifiers to generalize to each point in a time-shifted manner. Given the cross decoding nature of this approach, no cross-validation was performed, except for the testing on random (RD) tones, where a 5-fold cross validation, repeated five times, was implemented. For the sound-tosound and sound-to-omission decoding, time generalization was calculated for each entropy level separately, resulting in four generalization matrices, one for each entropy level. For each subject, 
481 depiction purposes, the training decoders weights were extracted and projected in the source space,

482 to localize the informative activity (see Figure 2b) related to carrier-frequency processing ${ }^{27,48}$.

\section{Statistical analysis}

As a first step, we extracted the dependence on entropy level within tinnitus and control groups. We arranged accuracy results for sounds from random to ordered and we then computed a regression for each single point of the testing-training $2 \mathrm{D}$ accuracy matrices, using the MATLAB built in least square mldivide algorithm (")"), resulting in a training time by testing time matrix of slopes (" $\beta$ ") for each subject, discarding intercepts. To compare the groups (25 Tinnitus subjects vs 25 age matched controls), we ran a t-test between the two matrices with coefficients obtained in the regression step, inputting them in the form of time-frequency 2D structures (timegeneralised $\beta$ values) in the $\mathrm{ft}$ _freqstatistics fieldtrip function. In order to account for multiple comparisons, we used a nonparametric cluster permutation test ${ }^{49}$, with 1000 permutations and a $p$ $<0.05$ to threshold the clusters.

We pursued further analysis with questionnaire data using $\mathrm{R}^{50}$. In the whole sample of participants with tinnitus (Tinnitus Ws, N=34) we performed a Spearman correlation of the $\beta$ coefficient values corresponding to the time-point of the maximum and the minimum t-value in intergroup analysis (comprised in positive and negative significant clusters emerging in group comparison for sound trials, see Figure 2c) with hearing loss (averaged audiogram for both ears), tinnitus loudness (10-point scale) and tinnitus distress scores (TQ). (see Supplementary Material Figure S5).

\section{Acknowledgements}

We thank Mr. Manfred Seifter for the help with the measurements and Miss Hayley Prins

507 for proofreading it.

508 This research is a part of the European School for Interdisciplinary Tinnitus Research project and

509 has received funding from the European Union's Horizon research and Innovation programme

510 under the Marie Sklodowska-Curie grant agreement number 722046. 


\section{References}

513 1. Shargorodsky, J., Curhan, G. C. \& Farwell, W. R. Prevalence and Characteristics of Tinnitus

$514 \quad$ among US Adults. Am. J. Med. 123, 711-718 (2010).

515 2. Bhatt, J. M., Lin, H. W. \& Bhattacharyya, N. Prevalence, Severity, Exposures, and Treatment

516 Patterns of Tinnitus in the United States. JAMA Otolaryngol.-- Head Neck Surg. 142, 959-965

$517 \quad(2016)$

518 3. Meyer, M., Luethi, M. S., Neff, P., Langer, N. \& Büchi, S. Disentangling Tinnitus Distress

519 and Tinnitus Presence by Means of EEG Power Analysis. Neural Plasticity

520 https://www.hindawi.com/journals/np/2014/468546/ (2014) doi:10.1155/2014/468546.

521 4. Schaette, R., Turtle, C. \& Munro, K. J. Reversible Induction of Phantom Auditory Sensations

522 through Simulated Unilateral Hearing Loss. PLOS ONE 7, e35238 (2012).

523 5. Weisz, N., Hartmann, T., Dohrmann, K., Schlee, W. \& Norena, A. High-frequency tinnitus

524 without hearing loss does not mean absence of deafferentation. Hear. Res. 222, 108-114

$525 \quad(2006)$.

526

527

6. Schaette, R. \& McAlpine, D. Tinnitus with a Normal Audiogram: Physiological Evidence for Hidden Hearing Loss and Computational Model. J. Neurosci. 31, 13452-13457 (2011).

7. Eggermont, J. J. \& Roberts, L. E. The neuroscience of tinnitus. Trends Neurosci. 27, 676-682 (2004).

8. De Ridder, D. et al. An integrative model of auditory phantom perception: Tinnitus as a

531 unified percept of interacting separable subnetworks. Neurosci. Biobehav. Rev. 44, 16-32 (2014).

9. Weisz, N. et al. The Neural Code of Auditory Phantom Perception. J. Neurosci. 27, 14791484 (2007).

535 10. Schaette, R. Tinnitus in men, mice (as well as other rodents), and machines. Hear. Res.

$536 \quad 311,63-71(2014)$. 
537 11. Roberts, L. E. \& Salvi, R. Overview: Hearing loss, tinnitus, hyperacusis, and the role of

$538 \quad$ central gain. Neuroscience (2019) doi:10.1016/j.neuroscience.2019.03.021.

539 12. Haegens, S., Nacher, V., Luna, R., Romo, R. \& Jensen, O. -Oscillations in the monkey

540 sensorimotor network influence discrimination performance by rhythmical inhibition of

541 neuronal spiking. Proc. Natl. Acad. Sci. 108, 19377-19382 (2011).

542 13. Leske, S. et al. The strength of alpha and beta oscillations parametrically scale with the 543 strength of an illusory auditory percept. NeuroImage 88, 69-78 (2014).

544 14. Müller, N. et al. You can't stop the music: Reduced auditory alpha power and coupling 545 between auditory and memory regions facilitate the illusory perception of music during noise.

$546 \quad$ NeuroImage 79, 383-393 (2013).

547 15. Elgoyhen, A. B., Langguth, B., De Ridder, D. \& Vanneste, S. Tinnitus: perspectives from 548 human neuroimaging. Nat. Rev. Neurosci. 16, 632-642 (2015).

549 16. Adjamian, P. The application of electro- and magneto-encephalography in tinnitus $550 \quad$ research - methods and interpretations. Front. Neurol. 5, 228 (2014).

551 17. Sedley, W. Tinnitus: Does Gain Explain? Neuroscience 407, 213-228 (2019).

552 18. Martines, F., Bentivegna, D., Martines, E., Sciacca, V. \& Martinciglio, G. Assessing 553 audiological, pathophysiological and psychological variables in tinnitus patients with or 554 without hearing loss. Eur. Arch. Otorhinolaryngol. 267, 1685-1693 (2010).

555 19. Goman, A. M. \& Lin, F. R. Prevalence of Hearing Loss by Severity in the United States. Am. J. Public Health 106, 1820-1822 (2016).

557 20. Nondahl, D. et al. Generational Differences in the Reporting of Tinnitus. Ear Hear. 33, $558 \quad 640-644(2012)$.

559 21. Sedley, W., Friston, K. J., Gander, P. E., Kumar, S. \& Griffiths, T. D. An Integrative 560 Tinnitus Model Based on Sensory Precision. Trends Neurosci. 39, 799-812 (2016).

561 22. Von Helmholtz, H. Handbuch der physiologischen Optik. vol. 9 (Voss, 1867). 
562 23. Sedley, W., Alter, K., Gander, P. E., Berger, J. \& Griffiths, T. D. Exposing pathological

563 sensory predictions in tinnitus using auditory intensity deviant evoked responses. J. Neurosci.

564 Off. J. Soc. Neurosci. (2019) doi:10.1523/JNEUROSCI.1308-19.2019.

565 24. Corlett, P. R. et al. Hallucinations and Strong Priors. Trends Cogn. Sci. (2018)

$566 \quad$ doi:10.1016/j.tics.2018.12.001.

567 25. Teufel, C. et al. Shift toward prior knowledge confers a perceptual advantage in early

568 psychosis and psychosis-prone healthy individuals. Proc. Natl. Acad. Sci. U. S. A. 112,

$569 \quad 13401-13406(2015)$.

570 26. Powers, A. R., Mathys, C. \& Corlett, P. R. Pavlovian conditioning-induced

571 hallucinations result from overweighting of perceptual priors. Science 357, 596-600 (2017).

572 27. Demarchi, G., Sanchez, G. \& Weisz, N. Automatic and feature-specific prediction-related

573 neural activity in the human auditory system. Nat. Commun. 10, 3440 (2019).

574 28. Kok, P., Mostert, P. \& de Lange, F. P. Prior expectations induce prestimulus sensory

575 templates. Proc. Natl. Acad. Sci. 114, 10473-10478 (2017).

576 29. Smith, F. W. \& Muckli, L. Nonstimulated early visual areas carry information about

577 surrounding context. Proc. Natl. Acad. Sci. U. S. A. 107, 20099-20103 (2010).

578 30. King, J.-R. \& Dehaene, S. Characterizing the dynamics of mental representations: the

579 temporal generalization method. Trends Cogn. Sci. 18, 203-210 (2014).

580 31. Rauschecker, J. P., Leaver, A. M. \& Mühlau, M. Tuning Out the Noise: Limbic-Auditory

581 Interactions in Tinnitus. Neuron 66, 819-826 (2010).

582 32. Thai-Van, H., Micheyl, C., Norena, A. \& Collet, L. Local improvement in auditory

583 frequency discrimination is associated with hearing-loss slope in subjects with cochlear

584 damage. Brain J. Neurol. 125, 524-537 (2002).

585 33. Langers, D. R. M., de Kleine, E. \& van Dijk, P. Tinnitus does not require macroscopic

586 tonotopic map reorganization. Front. Syst. Neurosci. 6, 2 (2012). 
587 34. Mühlnickel, W., Elbert, T., Taub, E. \& Flor, H. Reorganization of auditory cortex in 588 tinnitus. Proc. Natl. Acad. Sci. 95, 10340-10343 (1998).

$58935 . \quad$ Brima, T. et al. Auditory sensory memory span for duration is severely curtailed in $590 \quad$ females with Rett syndrome. Transl. Psychiatry 9, 130 (2019).

591 36. Ahlf, S., Tziridis, K., Korn, S., Strohmeyer, I. \& Schulze, H. Predisposition for and 592 Prevention of Subjective Tinnitus Development. PLoS ONE 7, e44519 (2012).

593 37. Goebel, G. \& Hiller, W. Psychische Beschwerden bei chronischem Tinnitus: Erprobung 594 und Evaluation des Tinnitus-Fragebogens (TF). Verhaltenstherapie 2, 13-22 (1992).

595 38. Newman, C. W., Jacobson, G. P. \& Spitzer, J. B. Development of the Tinnitus Handicap 596 Inventory. Arch. Otolaryngol. Head Neck Surg. 122, 143-148 (1996).

597 39. Meikle, M. B. et al. The tinnitus functional index: development of a new clinical 598 measure for chronic, intrusive tinnitus. Ear Hear. 33, 153-176 (2012).

599 40. Henry, J. A. et al. Tinnitus Functional Index: Development, validation, outcomes 600 research, and clinical application. Hear. Res. 334, 58-64 (2016).

601 41. Langguth, B. et al. Consensus for tinnitus patient assessment and treatment outcome 602 measurement: Tinnitus Research Initiative meeting, Regensburg, July 2006. Prog. Brain Res. $603 \quad \mathbf{1 6 6}, 525-536(2007)$.

604 42. Nastase, S., Iacovella, V. \& Hasson, U. Uncertainty in visual and auditory series is coded 605 by modality-general and modality-specific neural systems. Hum. Brain Mapp. 35, 1111-1128 $606 \quad$ (2014).

607 43. Brainard, D. H. The Psychophysics Toolbox. Spat Vis 10, 433-436 (1997).

608 44. Taulu, S. \& Kajola, M. Presentation of electromagnetic multichannel data: The signal 609 space separation method. J. Appl. Phys. 97, 124905 (2005).

610 45. Cichy, R. M. \& Pantazis, D. Multivariate pattern analysis of MEG and EEG: A 611 comparison of representational structure in time and space. NeuroImage 158, 441-454 (2017). 
612 46. Garcés, P., López-Sanz, D., Maestú, F. \& Pereda, E. Choice of magnetometers and

613 gradiometers after signal space separation. Sens. Switz. 17, 2926 (2017).

614 47. Oostenveld, R., Fries, P., Maris, E. \& Schoffelen, J. M. FieldTrip: Open source software

615 for advanced analysis of MEG, EEG, and invasive electrophysiological data. Comput. Intell.

$616 \quad$ Neurosci. 2011, (2011).

617 48. Marti, S. \& Dehaene, S. Discrete and continuous mechanisms of temporal selection in 618 rapid visual streams. Nat. Commun. 8, (2017).

619 49. Maris, E. \& Oostenveld, R. Nonparametric statistical testing of EEG-and MEG-data. $J$. $620 \quad$ Neurosci. Methods 164, 177-190 (2007).

621 50. Team, R. C. R: A language and environment for statistical computing. (2013).

622

623

624

625

626

627

628

629

630

631

632

633 
bioRxiv preprint doi: https://doi.org/10.1101/869842; this version posted December 10, 2019. The copyright holder for this preprint (which was not certified by peer review) is the author/funder, who has granted bioRxiv a license to display the preprint in perpetuity. It is made available under aCC-BY-NC-ND 4.0 International license. 
bioRxiv preprint doi: https://doi.org/10.1101/869842; this version posted December 10,2019 . The copyright holder for this preprint (which was not certified by peer review) is the author/funder, who has granted bioRxiv a license to display the preprint in perpetuity. It is made available under aCC-BY-NC-ND 4.0 International license.

\begin{tabular}{|c|c|c|c|c|c|c|c|}
\hline & & & total & $m$ & $f$ & & \\
\hline \multirow[t]{3}{*}{ Gender (n) } & Tinnitus Cmp & & 25 & 9 & 16 & & \\
\hline & Controls & & 25 & 8 & 17 & & \\
\hline & Tinnitus $W s$ & & 34 & 18 & 16 & & \\
\hline & & & $n$ & median & mean & $s d$ & \\
\hline \multirow[t]{3}{*}{ Age (years) } & Tinnitus Cmp & & 25 & 42 & 40.92 & 13.17 & \\
\hline & Controls & & 25 & 43 & 41.56 & 13.68 & \\
\hline & Tinnitus $W s$ & & 34 & 47.5 & 45.12 & 13.65 & \\
\hline \multicolumn{2}{|l|}{$\begin{array}{l}\text { Audiometry } \\
\text { (averaged thresholds, dB HL) }\end{array}$} & & $n$ & median & mean & $s d$ & distribution \\
\hline \multirow[t]{2}{*}{$125 \mathrm{~Hz}-2 \mathrm{kHz} \mathrm{R}$} & Tinnitus Cmp & & 23 & 14 & 16.91 & 11.58 & \\
\hline & Tinnitus $W_{S}$ & & 31 & 15 & 17.9 & 12.93 & . \\
\hline \multirow[t]{2}{*}{$125 \mathrm{~Hz}-2 \mathrm{kHz} \mathrm{L}$} & Tinnitus Cmp & & 23 & 12 & 12.65 & 7.02 & \\
\hline & Tinnitus $W s$ & & 31 & 12 & 12.81 & 7.01 & \\
\hline \multirow[t]{2}{*}{$125 \mathrm{~Hz}-2 \mathrm{kHz} \mathrm{R}+\mathrm{L}$} & Tinnitus Cmp & & 23 & 13.5 & 14.78 & 7.84 & \\
\hline & Tinnitus $W s$ & & 31 & 13.5 & 15.35 & 8.89 & \\
\hline \multirow[t]{2}{*}{$4 \mathrm{kHz}-8 \mathrm{kHz}$ R } & Tinnitus Cmp & & 23 & 13.33 & 18.26 & 18.69 & \\
\hline & Tinnitus $W_{S}$ & & 31 & 16.67 & 23.01 & 22.67 & \\
\hline \multirow[t]{2}{*}{$4 \mathrm{kHz}-8 \mathrm{kHz} \mathrm{L}$} & Tinnitus Cmp & & 23 & 15 & 18.62 & 17.96 & \\
\hline & Tinnitus $W s$ & & 31 & 15 & 21.99 & 19.35 & \\
\hline \multirow[t]{3}{*}{$4 \mathrm{kHz}-8 \mathrm{kHz}$ R+L } & Tinnitus Cmp & & 23 & 13.33 & 18.44 & 16.17 & \\
\hline & Tinnitus $W s$ & & 23 & 15 & 22.5 & 19.19 & \\
\hline & & $n$ & & median & mean & $s d$ & distribution \\
\hline \multirow[t]{2}{*}{ TQ (score) } & Tinnitus Cmp & 25 & & 27 & 30.24 & 25.78 & \\
\hline & Tinnitus Ws & 34 & & 27 & 29.5 & 17.53 & \\
\hline \multicolumn{8}{|l|}{ Tinnitus loudness } \\
\hline \multirow[t]{2}{*}{ (TS, 1-10 scale) } & Tinnitus Cmp & 24 & & 4.5 & 4.83 & 2.58 & \\
\hline & Tinnitus Ws & 33 & & 4 & 4.79 & 2.53 & \\
\hline
\end{tabular}

Table S1. Demographic characteristics of the subject sample and descriptive statistics for averaged hearing loss and tinnitus 639 characteristics in Tinnitus groups: Tinnitus Cmp for participants included in group comparison with Controls, Tinnitus Ws for the whole 641 and higher $(4 \mathrm{kHz}-8 \mathrm{kHz})$ frequency bands and presented here for right $(R)$ ), left $(L)$ and both ears $(R+L)$. Tinnitus distress scores 642 presented for Tinnitus Questionnaire (TQ). Tinnitus Loudness reported on the scale 1-10 from the Tinnitus Severity questionnaire. 


\begin{tabular}{|c|c|c|c|c|c|c|c|c|c|}
\hline Subject No & $\begin{array}{l}\text { Group } \\
\text { Comparison }\end{array}$ & $\begin{array}{l}125 \mathrm{~Hz} \\
R\end{array}$ & $\begin{array}{l}250 \mathrm{~Hz} \\
R\end{array}$ & $\begin{array}{l}500 \mathrm{~Hz} \\
R\end{array}$ & $\begin{array}{l}1 \mathrm{kHz} \\
R\end{array}$ & $\begin{array}{l}2 \mathrm{kHz} \\
R\end{array}$ & $\begin{array}{l}4 \mathrm{kHz} \\
R\end{array}$ & $\begin{array}{l}6 \mathrm{kHz} \\
R\end{array}$ & $\begin{array}{l}8 \mathrm{kHz} \\
R\end{array}$ \\
\hline 1 & Yes & 10 & 15 & 10 & 5 & 0 & 5 & 5 & 15 \\
\hline 2 & Yes & 5 & 5 & 5 & 5 & 5 & 0 & 0 & 5 \\
\hline 3 & Yes & -5 & 15 & 10 & 5 & 10 & 10 & 20 & 10 \\
\hline 4 & Yes & 5 & 5 & 5 & 5 & -5 & 0 & -5 & 10 \\
\hline 5 & Yes & 15 & 20 & 20 & 5 & 10 & 10 & 10 & 10 \\
\hline 6 & Yes & 15 & 15 & 10 & 10 & 5 & 5 & 10 & 5 \\
\hline 7 & Yes & $n a$ & $n a$ & na & na & $n a$ & $n a$ & $n a$ & $n a$ \\
\hline 8 & Yes & 5 & 5 & 15 & 10 & 10 & 5 & 5 & 5 \\
\hline 9 & Yes & $n a$ & $n a$ & na & na & $n a$ & $n a$ & $n a$ & $n a$ \\
\hline 10 & Yes & 25 & 25 & 25 & 15 & 15 & 10 & 20 & 20 \\
\hline 11 & Yes & 35 & 50 & 50 & 45 & 15 & 0 & 0 & 0 \\
\hline 12 & Yes & 25 & 15 & 15 & 10 & 10 & 5 & -10 & 0 \\
\hline 13 & Yes & 15 & 10 & 10 & 10 & 20 & 25 & 30 & 15 \\
\hline 14 & Yes & 10 & 10 & 10 & 10 & 0 & 15 & 20 & 30 \\
\hline 15 & Yes & 20 & 25 & 20 & 20 & 20 & 15 & 20 & 10 \\
\hline 16 & Yes & 20 & 25 & 25 & 35 & 30 & 30 & 25 & 10 \\
\hline 17 & Yes & 10 & 0 & 10 & 10 & 20 & 15 & 20 & 0 \\
\hline 18 & Yes & 45 & 65 & 55 & 55 & 50 & 55 & 75 & 80 \\
\hline 19 & Yes & 20 & 15 & 20 & 15 & 10 & 20 & 40 & 25 \\
\hline 20 & Yes & 30 & 25 & 15 & 10 & 10 & 0 & 5 & 5 \\
\hline 21 & Yes & 5 & 5 & 5 & 30 & 15 & 25 & 45 & 45 \\
\hline 22 & Yes & 25 & 25 & 20 & 5 & 0 & 10 & 20 & 15 \\
\hline 23 & Yes & 10 & 15 & 15 & 10 & 5 & 10 & 35 & 5 \\
\hline 24 & No & 20 & 20 & 20 & 20 & 20 & 55 & 60 & 45 \\
\hline 25 & Yes & 25 & 25 & 25 & 30 & 20 & 40 & 60 & 70 \\
\hline 26 & Yes & 15 & 15 & 25 & 15 & 10 & 15 & 35 & 15 \\
\hline 27 & No & 10 & 5 & 5 & 10 & 10 & 55 & 60 & 55 \\
\hline 28 & No & 25 & 25 & 25 & 20 & 20 & 20 & 15 & 20 \\
\hline 29 & No & $n a$ & $n a$ & $n a$ & $n a$ & $n a$ & $n a$ & $n a$ & $n a$ \\
\hline 30 & No & 30 & 30 & 20 & 20 & 30 & 40 & 55 & 60 \\
\hline 31 & No & 10 & 10 & 10 & 0 & 5 & 5 & 5 & -5 \\
\hline 32 & No & 25 & 25 & 20 & 20 & 25 & 20 & 25 & 30 \\
\hline 33 & No & 15 & 10 & 15 & 5 & 10 & 10 & 15 & 10 \\
\hline 34 & No & 50 & 55 & 60 & 60 & 70 & 80 & 90 & 100 \\
\hline
\end{tabular}

Table S2 a. Detailed audiograms (in $\mathrm{dB} \mathrm{HL}$ ) for each subject with tinnitus, right ear. 
bioRxiv preprint doi: https://doi.org/10.1101/869842; this version posted December 10, 2019. The copyright holder for this preprint (which was not certified by peer review) is the author/funder, who has granted bioRxiv a license to display the preprint in perpetuity. It is made available under aCC-BY-NC-ND 4.0 International license.

\begin{tabular}{|c|c|c|c|c|c|c|c|c|c|}
\hline Subject No & $\begin{array}{l}\text { Group } \\
\text { Comparison }\end{array}$ & $\begin{array}{l}125 \mathrm{~Hz} \\
L\end{array}$ & $\begin{array}{l}250 \mathrm{~Hz} \\
L\end{array}$ & $\begin{array}{l}500 \mathrm{~Hz} \\
L \\
\end{array}$ & $\begin{array}{l}1 \mathrm{kHz} \\
L\end{array}$ & $\begin{array}{l}2 k H z \\
L\end{array}$ & $\begin{array}{l}4 \mathrm{kHz} \\
L\end{array}$ & $\begin{array}{l}6 k \mathrm{kz} \\
L \\
\end{array}$ & $\begin{array}{l}8 \mathrm{kHz} \\
L\end{array}$ \\
\hline 1 & Yes & 0 & 5 & 5 & 0 & -5 & 0 & 0 & 0 \\
\hline 2 & Yes & 5 & 5 & 10 & 0 & 0 & 0 & -5 & 0 \\
\hline 3 & Yes & 5 & 10 & 10 & 10 & 0 & 10 & 10 & 15 \\
\hline 4 & Yes & 5 & 5 & 5 & 5 & 0 & 0 & -5 & -5 \\
\hline 5 & Yes & 25 & 25 & 20 & 15 & 10 & 10 & 15 & 10 \\
\hline 6 & Yes & 10 & 15 & 10 & 5 & 0 & 5 & 0 & 5 \\
\hline 7 & Yes & $n a$ & $n a$ & $n a$ & $n a$ & $n a$ & $n a$ & $n a$ & $n a$ \\
\hline 8 & Yes & 0 & 5 & 10 & 0 & 5 & 5 & 5 & 5 \\
\hline 9 & Yes & $n a$ & $n a$ & $n a$ & $n a$ & $n a$ & $n a$ & $n a$ & $n a$ \\
\hline 10 & Yes & 30 & 30 & 25 & 15 & 15 & 20 & 55 & 75 \\
\hline 11 & Yes & 40 & 10 & 10 & 5 & 5 & 10 & 15 & 25 \\
\hline 12 & Yes & 25 & 20 & 15 & 0 & 0 & 0 & -5 & 0 \\
\hline 13 & Yes & 15 & 10 & 15 & 10 & 10 & 5 & 25 & 20 \\
\hline 14 & Yes & 15 & 15 & 15 & 10 & 0 & 15 & 20 & 25 \\
\hline 15 & Yes & 20 & 20 & 20 & 20 & 20 & 10 & 15 & 20 \\
\hline 16 & Yes & 20 & 25 & 25 & 30 & 40 & 50 & 35 & 40 \\
\hline 17 & Yes & 5 & 5 & 10 & 15 & 25 & 10 & 20 & 15 \\
\hline 18 & Yes & 5 & 5 & 15 & 10 & 10 & 5 & 15 & 10 \\
\hline 19 & Yes & 20 & 15 & 25 & 25 & 15 & 30 & 35 & 25 \\
\hline 20 & Yes & 15 & 15 & 15 & 10 & 5 & 0 & 5 & 10 \\
\hline 21 & Yes & 10 & 5 & 5 & 0 & 10 & 25 & 35 & 55 \\
\hline 22 & Yes & 20 & 15 & 15 & 10 & 0 & 5 & 15 & 10 \\
\hline 23 & Yes & 5 & 5 & 5 & 10 & 10 & 10 & 5 & 15 \\
\hline 24 & No & 15 & 20 & 20 & 25 & 35 & 60 & 55 & 60 \\
\hline 25 & Yes & 20 & 10 & 10 & 10 & 10 & 20 & 40 & 80 \\
\hline 26 & Yes & 5 & 10 & 15 & 15 & 15 & 20 & 45 & 55 \\
\hline 27 & No & 10 & 10 & 5 & 5 & 5 & 40 & 60 & 55 \\
\hline 28 & No & 20 & 20 & 20 & 10 & 5 & 5 & 5 & 10 \\
\hline 29 & No & $n a$ & $n a$ & $n a$ & $n a$ & $n a$ & $n a$ & $n a$ & $n a$ \\
\hline 30 & No & 25 & 20 & 15 & 15 & 35 & 50 & 60 & 60 \\
\hline 31 & No & 5 & 5 & 10 & 10 & 0 & 5 & 5 & 5 \\
\hline 32 & No & 25 & 25 & 20 & 10 & 5 & 20 & 30 & 35 \\
\hline 33 & No & 10 & 15 & 10 & 10 & 15 & 30 & 35 & 55 \\
\hline 34 & No & 30 & 30 & 30 & 20 & 20 & 35 & 45 & 55 \\
\hline
\end{tabular}

650 Table S2 b. Detailed audiograms (in $\mathrm{dB} \mathrm{HL}$ ) for each subject with tinnitus, left ear. 
bioRxiv preprint doi: https://doi.org/10.1101/869842; this version posted December 10, 2019. The copyright holder for this preprint (which was not certified by peer review) is the author/funder, who has granted bioRxiv a license to display the preprint in perpetuity. It is made available under aCC-BY-NC-ND 4.0 International license.

\section{Time-generalised regression $\beta$}

a)

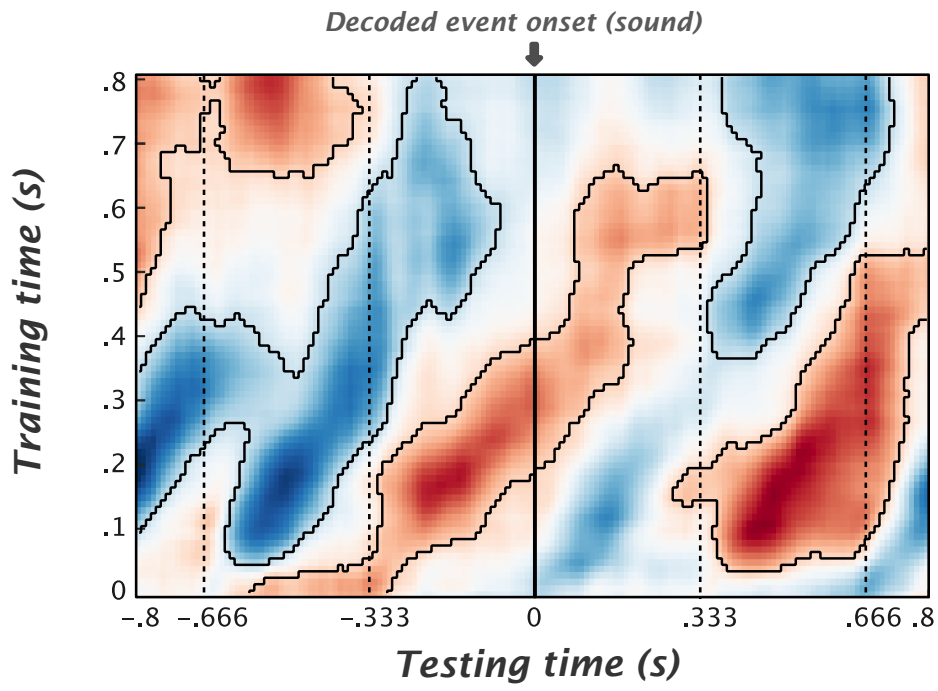

b)

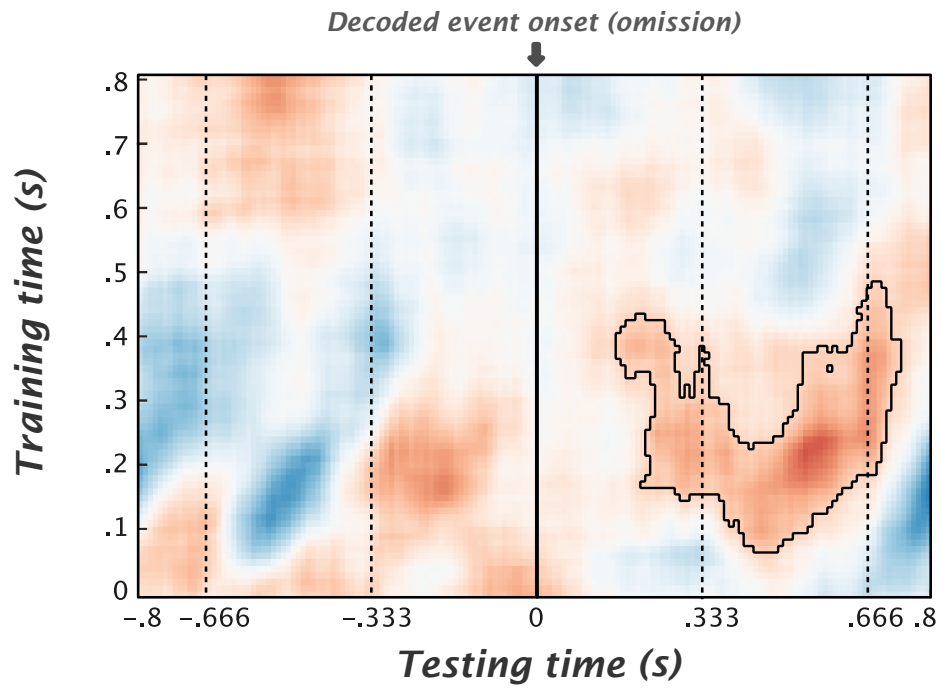

Figure S3. Time generalisation of $\beta$-coefficient values in the sample of Tinnitus $\mathrm{Cmp}$ and Controls joint together ( $\mathrm{N}=50)$, tested against 0: a) For sound trials, we observe a pattern as reported in the previous study: carrier frequency specific templates are most strongly driven by early training time ( $100-150 \mathrm{~ms})$ and emerge in accordance to regularity level, in anticipatory period before the presentation of the sound as well as after the presentation of the consecutive tone ( $450 \mathrm{~ms}$ in testing time). In the omission trials (b) the pre-stimulus effects do not reach significance but we observe the post-stimulus significant linear increase of decoding accuracy with regularity, emerging at approx $\sim 150 \mathrm{~ms}$ after expected sound omission. These results point to presence of anticipatory activation of the templates corresponding to carrier frequency dependent on predictability and reactivation based on the knowledge about the sound sequence, putatively related to short-term memory processes. 
a)

\section{Time-generalised regression $\beta$ Tinnitus vs. Control}

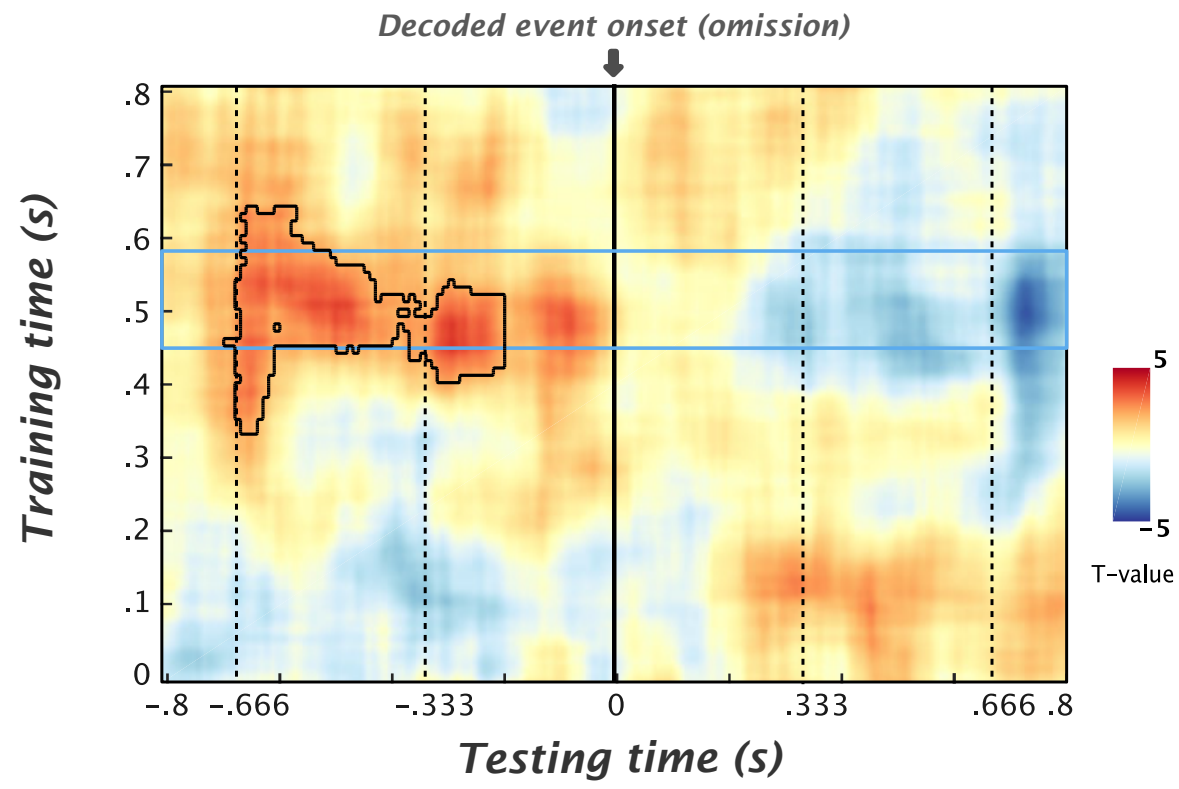

b)

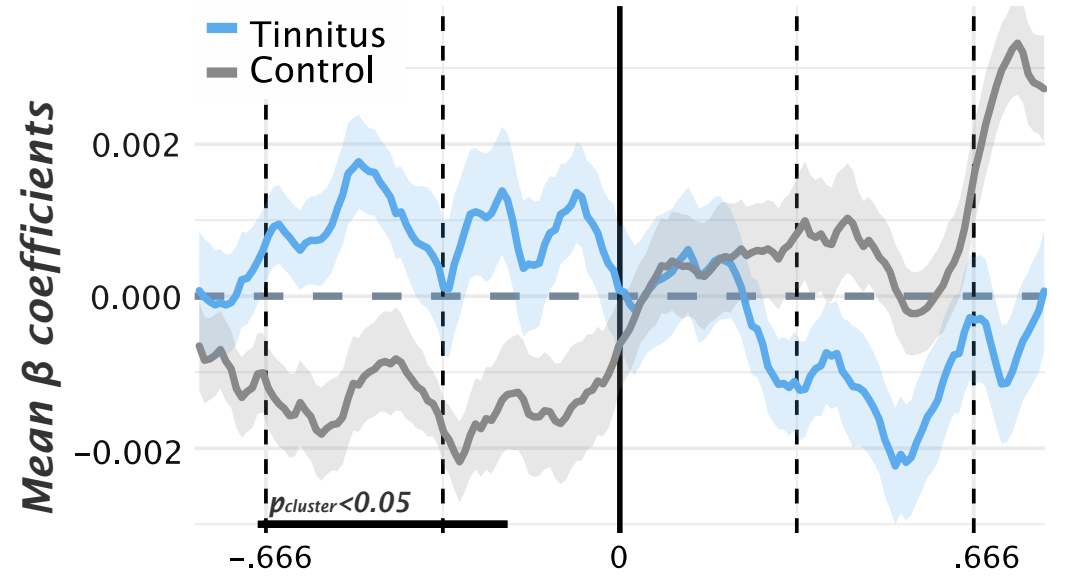

Testing time (s)

Figure S4. a) Group comparison (see Figure 1c) of $\beta$-coefficient values between Tinnitus vs. Control groups in time-generalised matrix in omission trials. Colors indicate t-values and solid black borders delimiting periods of significant difference $(p<0.05$, cluster corrected). b) Time courses of $\beta$-coefficients averaged over $480-580 \mathrm{~ms}$ training time-window (indicated by the blue rectangle and corresponding to the one previously demonstrated in sound-type trials), showing effects driven by a relative increase of regularitydependent carrier frequency specific activity prior to anticipated onset period in Tinnitus group. 
bioRxiv preprint doi: https://doi.org/10.1101/869842; this version posted December 10, 2019. The copyright holder for this preprint (which was not certified by peer review) is the author/funder, who has granted bioRxiv a license to display the preprint in perpetuity. It is made available under aCC-BY-NC-ND 4.0 International license.

681

682

683

684 a)

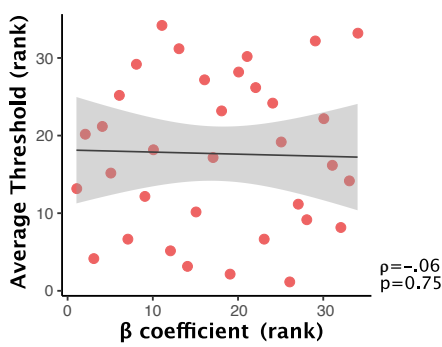

b)

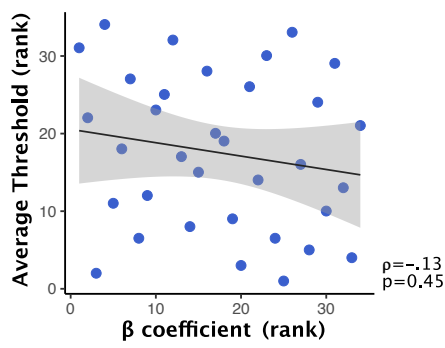

Tinnitus loudness

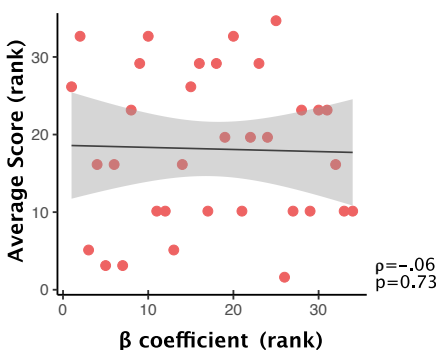

Tinnitus loudness

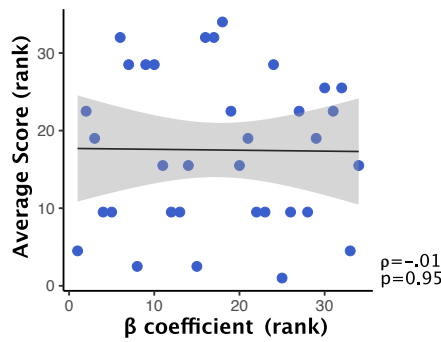

Tinnitus distress

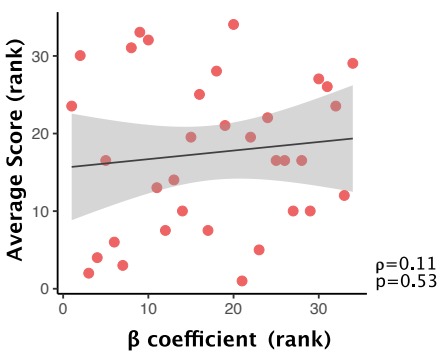

Tinnitus distress

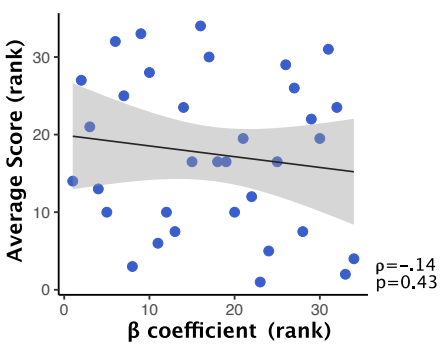

685

686

687

688

689

690

691

692
Figure S5. Scatter plots of hearing loss (left), tinnitus loudness (1-10 scale, middle) and tinnitus distress (TQ, right) measures with individual $\beta$-coefficient values (same as in Figure 2c) in Tinnitus group. a.) Pre-stimulus positive cluster, no significant correlation was revealed ( $p>0.05$, uncorrected). b) Post-stimulus negative cluster, no significant correlation was shown for any of the tested factors ( $p>0.05$, uncorrected). 